TRANSACTIONS OF THE

AMERICAN MATHEMATICAL SOCIETY

Volume 356, Number 1, Pages 393-414

S 0002-9947(03)03352-X

Article electronically published on August 21, 2003

\title{
ASYMPTOTICS OF THE TRANSITION PROBABILITIES OF THE SIMPLE RANDOM WALK ON SELF-SIMILAR GRAPHS
}

\author{
BERNHARD KRÖN AND ELMAR TEUFL
}

\begin{abstract}
It is shown explicitly how self-similar graphs can be obtained as 'blow-up' constructions of finite cell graphs $\hat{C}$. This yields a larger family of graphs than the graphs obtained by discretising continuous self-similar fractals.

For a class of symmetrically self-similar graphs we study the simple random walk on a cell graph $\hat{C}$, starting at a vertex $v$ of the boundary of $\hat{C}$. It is proved that the expected number of returns to $v$ before hitting another vertex in the boundary coincides with the resistance scaling factor.

Using techniques from complex rational iteration and singularity analysis for Green functions, we compute the asymptotic behaviour of the $n$-step transition probabilities of the simple random walk on the whole graph. The results of Grabner and Woess for the Sierpiński graph are generalised to the class of symmetrically self-similar graphs, and at the same time the error term of the asymptotic expression is improved. Finally, we present a criterion for the occurrence of oscillating phenomena of the $n$-step transition probabilities.
\end{abstract}

\section{INTRODUCTION}

Self-similar fractals are usually constructed as the compact invariant set of an iterated function system. Analysis on these sets is still a rapidly growing field in mathematics. One approach is to study self-similar graphs as the discretization of fractals and then transfer the results on these graphs back to the fractals via rescaling. In this way one can construct Brownian motion on fractals by starting with random walks on graphs; see for example Barlow [2], Lindstrøm [20] and the references therein.

Contracted images of a fractal as compact subsets of the fractal are called cells. Correspondingly, self-similar graphs have cell graphs as finite subgraphs which carry essential information about the whole graph. Malozemov and Teplyaev defined self-similarity of graphs axiomatically in [21]. Their definition was restricted to the case where the cells of the graph have exactly two boundary points. In [18] one of the present authors introduced self-similarity for an arbitrary number of boundary points. Another axiomatic approach for self-similar graphs with more boundary points was chosen in [22]. Sabot constructed self-similar graphs using equivalence relations on word spaces; see [26].

Received by the editors August 19, 2002 and, in revised form, March 9, 2003.

2000 Mathematics Subject Classification. Primary 60J10; Secondary 05A15, 30D05.

Key words and phrases. Self-similar graphs, simple random walk, transition probability.

The first author was supported by the project P14379-MAT of the Austrian Science Fund (FWF).

The second author was supported by the START-project Y96-MAT of the FWF. 
Probably the most extensively studied self-similar graph is the Sierpiński graph. In [12] Grabner and Woess considered the Green function of the simple random walk on the Sierpiński graph which describes the returning of the random walk to a certain origin vertex. A combinatorial substitution based on path arguments was used to obtain a functional equation for this Green function. Iterating this equation, they obtained the analytic continuation of the Green function as a rapidly converging product of rational terms. The asymptotic behaviour and oscillation phenomena were computed by analysing the singularity of the Green function at $z=1$. These techniques had been introduced by Flajolet and Odlyzko in 8 and [23]. Symmetrically self-similar graphs were constructed in [18] as a class of selfsimilar graphs such that the combinatorial substitution mentioned above can be applied. Sections 5 to 7 of the present paper are a generalisation and a further development of the asymptotic analysis in [12].

In Section 2 we recall from [18 the axiomatic definition of self-similarity of graphs and a graph-theoretic analogue to the Banach fixed point theorem for self-similar graphs. Heuristically, this theorem says that a self-similar graph has a "centre", either as an origin vertex or as an origin cell. We will later focus on self-similar graphs with an origin vertex. Our axiomatic approach to self-similarity of graphs is based on contracting a given infinite graph. On the other hand, one can obtain self-similar graphs by a "blow-up" construction, starting with a finite cell graph. Theorem 1 yields this construction explicitly; the resulting graphs may have more irregular structures than discretized post-critically finite self-similar (pcfss) sets; see 2, 15] for the notion of pcfss sets. It is proved that a symmetrically self-similar graph is bipartite if and only if its cell graphs are bipartite.

Section 3 is devoted to the study of Green functions. For the Sierpiński graph, a functional equation

$$
G(z)=f(z) G(d(z))
$$

is valid for the Green function $G$ at the origin vertex, which was first observed by Rammal and Toulouse [24, 25]; see also [12]. In [18] this equation was generalised to the class of so-called symmetrically self-similar graphs. One property of these graphs is that their cell graphs are all isomorphic. The functions $d$ and $f$ are rational functions associated with the simple random walk on the cell graph $\hat{C}$. The transition function $d$ is the generating function of the probabilities that the simple random walk, starting at the boundary of $\hat{C}$, hits another vertex in the boundary for the first time after exactly $n$ steps. The product

$$
\prod_{k=0}^{\infty} f\left(d^{k}(z)\right)
$$

converges on the Fatou set $\mathcal{F}$ of $d$ (possibly with the exception of countably many points) to the Green function $G$ as a solution of the functional equation above. The point $z=0$ is the only attracting fixed point of $d$, and the Fatou set $\mathcal{F}$ contains $(\mathbb{C} \backslash \mathbb{R}) \cup(-1,1)$.

The simple random walk on a cell graph $\hat{C}$ of a symmetrically self-similar graph $X$ is studied in Section 4 The number $\theta$ of vertices in the boundary of a cell graph does not depend on the choice of the cell. The graph $\hat{C}$ consists of $\mu$ amalgamated copies of the $\theta$-complete graph $K_{\theta}$. This parameter $\mu$ corresponds to the usual mass scaling factor of self-similar sets. The number $\tau=d^{\prime}(1)$ is called the time scaling factor. Let this random walk start at a vertex $v$ in the boundary of $C$. Then $\tau$ is 
the average time until hitting a boundary vertex other than $v$. For symmetrically self-similar graphs with bounded geometry it is proved that $f(1)=\rho$, where $f(1)$ is the expected number of returns to $v$ before hitting a new vertex in the boundary of $\hat{C}$ and $\rho$ is the resistance scaling factor; see [2]. For the proof we use the relation $\tau=\mu \rho$.

In Section 5 we study the asymptotic behaviour of the $n$-step return probabilities. Using the functional equation of Section 3, we derive a local singular expansion of $G$ in $z=1$,

$$
G(z)=(1-z)^{\frac{\log (\mu)}{\log (\tau)}-1}\left(\omega\left(\frac{\log (1-z)}{\log (\tau)}\right)+\mathcal{O}_{\delta}(|z-1|)\right)
$$

for all $z \in \mathbb{C}$ such that $|z-1|<r$ and $|\arg (1-z)|<\delta$, where $0<\delta<\pi$ and $r>0$. The function $\omega$ is 1-periodic and holomorphic on some horizontal strip around the real axis. In [12] this type of expansion was computed for the Sierpiński graph with a weaker error term. Using the method of singularity analysis, we obtain the asymptotics of the $n$-step return probabilities $p^{(n)}(o, o)$ to an origin vertex $o$. For the case that $X$ is not bipartite we have

$$
p^{(n)}(o, o)=n^{-\frac{\log (\mu)}{\log (\tau)}}\left(\sigma\left(\frac{\log (n)}{\log (\tau)}\right)+\mathcal{O}\left(n^{-1}\right)\right),
$$

where $\sigma$ is a 1-periodic, holomorphic function defined on some horizontal strip around the real axis. If $X$ is bipartite, then $p^{(2 n+1)}(o, o)=0$ and $p^{(2 n)}(o, o)$ satisfies the above asymptotic expansion. This type of oscillation seems to be typical for random walks on self-similar graphs and Brownian motion on fractals under symmetry conditions; see [3, 5, 9, 11, 12, 17, 27].

It is proved in Section [6 that the function $\sigma$ is not constant if the Julia set $\mathcal{J}$ of $d$ is a Cantor set.

In Section 7 it is shown that for any pair of vertices $x$ and $y$, the transition probabilities $p^{(n)}(x, y)$ satisfy the same asymptotic expansion as $p^{(n)}(o, o)$. For this purpose we use a ratio limit theorem; see [12].

Section 8 is devoted to the case where the cell graph is a finite line $L_{n}$ of length $n$. The corresponding transition functions $d_{n}$ are conjugated to the Chebyshev polynomials $T_{n}$ via the conjugacy map $z \mapsto \frac{1}{z}$. Their Julia set is $(\mathbb{R} \cup\{\infty\}) \backslash(-1,1)$, and the periodic function $\sigma$ is constant. We study the probability for hitting a given vertex in the minimal number of steps for general, locally finite, reversible Markov chains. This probability is maximal whenever the process can be projected on the simple random walk on $L_{n}$. From this we deduce that the cell-graphs of symmetrically self-similar graphs are isomorphic to $L_{n}$ if and only if the transition function is conjugated to $T_{n}$ in the sense above. We are interested in characterisations of this type because we believe that these are the only symmetrically self-similar graphs with a constant periodic function $\sigma$. This would mean that in all other cases we may observe a non-trivial oscillation of the asymptotic transition probabilities.

In Section 9 some examples are discussed.

\section{SELF-SIMILAR GRAPHS}

Graphs $X=(V X, E X)$ with vertex set $V X$ and edge set $E X$ are always supposed to be undirected, without loops or multiple edges. Vertices $x$ and $y$ are adjacent if $\{x, y\}$ is an edge in $E X$. The degree $\operatorname{deg}_{X}(x)$ of $x$ is the number of vertices in $V X$ that are adjacent to $x$. A graph has bounded geometry if the set of vertex degrees is 
bounded. The vertex boundary or boundary $\theta C$ of a set $C$ of vertices in $V X$ is the set of vertices in $V X \backslash C$ which are adjacent to some vertex in $C$. A path of length $n \in \mathbb{N}_{0}$ from a vertex $v$ to a vertex $w$ is an $(n+1)$-tuple $\pi=\left(v=x_{0}, x_{1}, \ldots, x_{n}=w\right)$ of vertices such that $x_{i-1}$ and $x_{i}$ are adjacent for $1 \leq i \leq n$. The path $\pi$ is closed if $v=w$. The distance $d_{X}(v, w)$ between $v$ and $w$ in $X$ is the length of the shortest path from $v$ to $w$. A set $C$ of vertices is called connected if any pair of vertices in $C$ can be connected by a path in $X$ which does not leave $C$. We write $\hat{C}$ for the subgraph of $X$ spanned by $C \cup \theta C$.

We briefly repeat the definition of self-similar graphs (see Definitions 1 and 2 in [18 or in [19]): Let $F$ be a set of vertices in $V X$ and let $\mathcal{C}_{X}(F)$ be the set of connected components in $V X \backslash F$. We define the reduced graph $X_{F}$ of $X$ by setting $V X_{F}=F$ and connecting two vertices $x$ and $y$ in $V X_{F}$ by an edge if and only if there exists a component $C$ in $\mathcal{C}_{X}(F)$ such that $x$ and $y$ are in the boundary of $C$.

Definition 1. A connected infinite graph $X$ is called self-similar with respect to $F \subset V X$ and $\psi: V X \rightarrow V X_{F}$ if

(F1) no vertices in $F$ are adjacent in $X$,

(F2) the intersection of the boundaries of two different components in $\mathcal{C}_{X}(F)$ contains not more than one vertex, and

(F3) $\psi$ is an isomorphism between $X$ and $X_{F}$.

We will write $\phi$ instead of $\psi^{-1}$ and $F^{n}$ instead of $\psi^{n}(F)$, where $\psi^{n}$ denotes the $n$-fold iterate of $\psi$. Components of $\mathcal{C}_{X}\left(F^{n}\right)$ are $n$-cells; 1 -cells are also just called cells. An origin cell is a cell $C$ such that $\phi(\theta C) \subset C$. A fixed point of $\psi$ is called an origin vertex. See Section 9 for examples.

The following lemma is a graph-theoretic analogue to the Banach fixed point theorem; see [18, Theorem 1] or [19, Lemma 1].

Lemma 1. Let $X$ be self-similar with respect to $F$ and $\psi$. Then $X$ is self-similar with respect to $F^{k}$ and $\psi^{k}$, for any positive integer $k$. The graph $X$ has either

(a) exactly one origin cell and no origin vertex, or

(b) exactly one origin vertex $o$. If in this case $X$ is locally finite, then there is a positive integer $n$ such that the subgraphs $X_{A}$ of $X$ which are spanned by $A \cup\{o\}$, for components $A$ in $\mathcal{C}_{X}(\{o\})$, are self-similar graphs with respect to

$$
F_{A}=\{o\} \cup\left(F^{n} \cap A\right) \quad \text { and } \quad \psi_{A}=\left.\psi^{n}\right|_{F_{A}} .
$$

The graphs $X_{A}$ have exactly one origin cell, and o is their origin vertex.

Definition 2. Let $X$ be a graph which is self-similar with respect to $F$ and $\psi$. We call $\hat{C}_{n}$ the $n$-cell graph $C_{n}$; 1-cell graphs are also called cell graphs. The graph $X$ is symmetric (or doubly symmetric) if it is locally finite and satisfies the following axioms:

(S1) All cells are finite, and for any pair of cells $C$ and $D$ in $\mathcal{C}_{X}(F)$ there exists an isomorphism $\alpha: V \hat{C} \rightarrow V \hat{D}$ of $\hat{C}$ and $\hat{D}$ such that $\alpha(\theta C)=\theta D$.

(S2) The automorphism group $\operatorname{Aut}(\hat{C})$ of $\hat{C}$ acts doubly transitive on $\theta C$, which means that it acts transitively on the set

$$
\{(x, y) \mid x, y \in \theta C, x \neq y\},
$$

where $g((x, y))$ is defined as $(g(x), g(y))$ for any $g \in \operatorname{Aut}(\hat{C})$. 
If a self-similar graph $X$ satisfies (S1), then let $\theta$ be the number of vertices in the boundary of a cell. Axiom (S1) implies that $\theta$ is independent of the choice of the cell $C$.

The next theorem describes a 'blow-up' construction for self-similar graphs satisfying Axiom (S1).

Theorem 1. Let $G=(V G, E G)$ be a finite, connected graph and let $B$ be a subset of $V G$ with the following properties:

- The set $B$ has at least two elements, $V G \backslash B$ is connected, and no pair of vertices in $B$ is adjacent in $G$.

- The graph $G$ consists of $\mu$ complete subgraphs $K_{\theta}^{1}, \ldots, K_{\theta}^{\mu}$ which are isomorphic to the complete graph $K_{\theta}$ with $\theta$ vertices, such that $\theta=|B|$ and any two of these complete subgraphs have at most one vertex in common.

Then for each $i$ in $\{1, \ldots, \mu\}$ there are a graph $X$, a set $F \subset V X$ and a map $\psi: V X \rightarrow V X_{F}$ such that $X$ is self-similar with respect to $F$ and $\psi$, and the following statements are true:

(a) The set $V G \backslash B$ is the unique origin cell of $X, G$ is the corresponding cell graph, and $\psi\left(V K_{\theta}^{i}\right)=B$.

(b) For any cell $C$ there exists an isomorphism $\alpha: V \hat{C} \rightarrow V G$ of $\hat{C}$ and $G$ such that $\alpha(\theta C)=B$.

(c) A vertex $o$ is the origin vertex of $X$ if and only if it is a fixed point of $\psi$ in $V K_{\theta}^{i}$. In this case $V K_{\theta}^{i} \cap B=\{o\}$ and $V X \backslash\{o\}$ is connected.

Proof. We construct sequences of graphs $\left(Y_{n}\right)_{n \in \mathbb{N}}$, sets of vertices $\left(F_{n}\right)_{n \in \mathbb{N}}$, where $F_{n} \subset V Y_{n}$, and functions $\left(\psi_{n}\right)_{n \in \mathbb{N}}$ inductively such that they satisfy the following:

- The function $\psi_{n}$ is an isomorphism between $Y_{n-1}$ and the reduced graph $\left(Y_{n}\right)_{F_{n}}$.

- The graph $X=\bigcup_{n=1}^{\infty} Y_{n}$ (seen as union of graphs and not as set-theoretic union) is self-similar with respect to $F=\bigcup_{n=1}^{\infty} F_{n}$ and $\psi: V X \rightarrow F$, where $\psi$ is defined by $\left.\psi\right|_{V Y_{n}}=\psi_{n+1}$.

- $X$ has the properties required in the theorem.

First we set $V Y_{-1}=\emptyset, Y_{0}=K_{\theta}^{i}, Y_{1}=G$ and $F_{1}=B$. Let $\psi_{1}: V Y_{0} \rightarrow F_{1}$ be any bijective function. We suppose that there are graphs $Y_{k}$ and sets of vertices $F_{k} \subset V Y_{k}$, for $1 \leq k \leq n$, such that $Y_{k-1}$ is a subgraph of $Y_{k}$ and there is an isomorphism $\psi_{k}: V Y_{k-1} \rightarrow F_{k}$ of $Y_{k-1}$ and $\left(Y_{k}\right)_{F_{k}}$.

Let $\tilde{Y}_{n}$ be a disjoint, isomorphic copy of $Y_{n}$ and let $\alpha_{n}: V Y_{n} \rightarrow V \tilde{Y}_{n}$ be the corresponding isomorphism. In $\tilde{Y}_{n}$ the image $\alpha_{n}\left(Y_{n-1}\right)$ is now replaced by $Y_{n}$ such that for any $x \in V Y_{0}$ the vertex $\psi_{n} \circ \cdots \circ \psi_{1}(x)$ is identified with

$$
\alpha_{n}\left(\psi_{n-1} \circ \cdots \circ \psi_{1}(x)\right) .
$$

In the resulting graph we replace every $\theta$-complete graph $H$ that is not completely contained in $Y_{n}$ by an isomorphic copy of $G$ such that any different vertices in the copy that correspond to vertices in $B$ are amalgamated with different vertices in $V H$. In general this procedure of replacing the $\theta$-complete graphs is not unique. Let $Y_{n+1}$ be the resulting graph.

We set $F_{n+1}=F_{n} \cup \alpha_{n}\left(V Y_{n} \backslash V Y_{n-1}\right)$. The function

$$
\psi_{n+1}: V Y_{n} \rightarrow F_{n+1}
$$


defined by

$$
\left.\psi_{n+1}\right|_{V Y_{n-1}}=\psi_{n} \quad \text { and }\left.\quad \psi_{n+1}\right|_{V Y_{n} \backslash V Y_{n-1}}=\left.\alpha_{n}\right|_{V Y_{n} \backslash V Y_{n-1}}
$$

is an isomorphism between $Y_{n}$ and $\left(Y_{n+1}\right)_{F_{n+1}}$. The sequences $\left(Y_{n}\right)_{n \in \mathbb{N}}$ and $\left(F_{n}\right)_{n \in \mathbb{N}}$ are increasing, and $X=\bigcup_{n=1}^{\infty} Y_{n}$ is self-similar with respect to $F=\bigcup_{n=1}^{\infty} F_{n}$ and $\psi: V X \rightarrow F$ defined by $\left.\psi\right|_{V Y_{n}}=\psi_{n+1}$.

This construction implies that (a) and (b) are satisfied. We have

$$
d_{X}(\psi(x), \psi(y)) \geq 2 d_{X}(x, y)
$$

for the 'blow-up' function $\psi$ and vertices $x$ and $y$ in $V X$, and

$$
d_{X}(\phi(v), \phi(w)) \leq \frac{1}{2} d_{X}(v, w)
$$

for its inverse contraction $\phi=\psi^{-1}$ which is defined on $F$; for details see [18. Theorem 1]. This implies that a fixed point $o$ must lie in $V K_{\theta}^{i} \cap B$, and this intersection is a singleton. The set $\theta\{o\}=V K_{\theta}^{i} \backslash\{o\}$ is connected in $X$; thus $V X \backslash\{o\}$ is connected.

By choosing $\theta=2$ every connected finite graph which is not complete can occur as cell graph of a self-similar graph satisfying (S1). Any self-similar graph with a unique origin cell can be obtained as a blow-up in the sense of Theorem 1; thus it characterises self-similar graphs which remain connected by removing an origin vertex (see Lemma 1).

Let $X$ be a symmetrically self-similar graph and let $C$ be any 1-cell of $X$. Then there exists exactly one 2-cell $C_{2}$ such that $\psi(C) \subset C_{2}$. We denote the 1-cells contained in $C_{2}$ by $C^{1}, \ldots, C^{\mu}$. The cell graph $\hat{C}$ consists of $\mu$ amalgamated $\theta$ complete graphs $K_{\theta}^{1}, \ldots, K_{\theta}^{\mu}$, where $V K_{\theta}^{i}=\phi\left(\theta C^{i}\right)$ and $i \in\{1, \ldots, \mu\}$; see [19. For a vertex $v \in V \hat{C}$ we call

$$
\beta(v)=\left|\left\{i \in\{1, \ldots, \mu\} \mid v \in V K_{\theta}^{i}\right\}\right|
$$

the branching number of $v$. By Axiom (S2), Aut $(\hat{C})$ acts transitively on $\theta C$. Thus all vertices $v$ of the boundary $\theta C$ have the same branching number $\beta(v)$. Furthermore, Axiom (S1) implies that all cell graphs are isomorphic and $\beta(v)$ also does not depend on the choice of the cell $C$. Consequently, we define the branching number $\beta$ of a symmetrically self-similar graph as the branching number $\beta(v)$ of any vertex $v$ in $F$. This branching number characterises symmetrically self-similar graphs with bounded geometry:

Theorem 2. A symmetrically self-similar graph $X$ has bounded geometry if and only if $\beta=1$.

This theorem is an immediate consequence of Theorem 3 in [19. The next theorem is a special case of Corollary 1 in [19].

Theorem 3. Let $X$ be a symmetrically self-similar graph with unbounded geometry. Then either there exists no origin vertex and $X$ is locally finite, or there is an origin vertex which is then the only vertex with infinite degree.

In the following lemma we characterise those self-similar graphs satisfying (S1) which are bipartite.

Lemma 2. Let $X$ be a self-similar graph with respect to $F$ and $\psi$ satisfying $A x$ iom (S1). Then $X$ is bipartite if and only if some cell graph is bipartite. Furthermore, we have $\theta=2$ if $X$ is bipartite. 
Proof. If $X$ is bipartite, then every cell graph is bipartite, since it is a subgraph of $X$.

Now suppose that some cell graph is bipartite, whence all cell graphs are bipartite thanks to Axiom (S1). A $\theta$-complete graph is bipartite if and only if $\theta \leq 2$. Any cell graph $\hat{C}$ consists of $\mu$ amalgamated copies of $\theta$-complete graphs; hence $\theta=2$. Every closed path in $\hat{C}$ has even length. Either all paths in $\hat{C}$ connecting the two vertices in $\theta C$ have even length, or they all have odd length.

Suppose that $X$ is not bipartite. Then there exists a closed path $\pi$ in $X$ with minimal odd length. This path cannot be completely contained in one cell graph; hence it meets vertices in $F$. Let $\pi=\left(x_{0}, \ldots, x_{n}\right)$ with $x_{0}=x_{n} \in F$ and $x_{k+n}=x_{k}$ for all $k \in \mathbb{N}$. Let $x_{i}, x_{j}$ be vertices in $F$ such that $i<j$ and $x_{k} \notin F$ for all $i<k<j$. Then $j<i+n$, since $\pi$ is not contained in one cell graph. Moreover, we have $x_{i} \neq x_{j}$. Otherwise $\left(x_{i}, \ldots, x_{j}\right)$ would be a closed path in one cell graph and $\left(x_{j}, \ldots, x_{i+n}\right)$ would be a closed path in $X$ with odd length strictly smaller than $n$. Let $0=i_{0}<\cdots<i_{\ell}=n$ be all those indices such that $x_{i_{k}} \in F$ for $k \in\{0, \ldots, \ell\}$. Since the lengths of the paths $\left(x_{i_{k-1}}, \ldots, x_{i_{k}}\right)$ for $k \in\{1, \ldots, \ell\}$ have the same parity, $\left(\phi\left(x_{i_{0}}\right), \ldots, \phi\left(x_{i_{\ell}}\right)\right)$ is a closed path in $X$ of odd length strictly smaller than $n$, in contradiction to the minimality of $\pi$.

\section{Green function at the origin}

In this section we repeat and reformulate results and definitions from [18. Let

$$
P=(p(x, y))_{x, y \in V X}
$$

be the matrix of transition probabilities for the simple random walk on a symmetrically self-similar graph $X$ which is locally finite. The Green function for vertices $x$ and $y$ in $V X$ is defined as the generating function

$$
G(x, y \mid z)=\sum_{n=0}^{\infty} p^{(n)}(x, y) z^{n}
$$

of the $n$-step transition probabilities $p^{(n)}(x, y)$ from $x$ to $y$ for $z \in U(0,1)$, where $U\left(z_{0}, r\right)=\left\{z \in \mathbb{C}|| z-z_{0} \mid<r\right\}$ for any $z_{0} \in \mathbb{C}$ and $r>0$. If the Green function is considered as an infinite dimensional matrix, we have

$$
(G(x, y \mid z))_{x, y \in V X}=\sum_{n=0}^{\infty} P^{n} z^{n}=(I-z P)^{-1} .
$$

Let $C$ be a cell and let $B$ be a nonempty subset of $\theta C$. Then

$$
Q_{B}=\left(q_{B}(x, y)\right)_{x, y \in V \hat{C}}
$$

denotes the transition matrix of the simple random walk on $\hat{C}$ with absorbing boundary $B$. This means that

$$
q_{B}(x, y)=\frac{1}{\operatorname{deg}_{\hat{C}}(x)}
$$

if $x \in V \hat{C} \backslash B$ is adjacent to a vertex $y$ in $V \hat{C}$, and $q(x, y)=0$ otherwise. We define

$$
Q_{B}^{*}(z)=\sum_{n=0}^{\infty}\left(z Q_{B}\right)^{n}=\left(I-z Q_{B}\right)^{-1}
$$


for all $z \in \mathbb{C}$ such that $I-z Q_{B}$ is invertible. The entries of the matrix $Q_{B}^{*}(z)$ are rational functions in $z$ which are holomorphic on $U(0, r)$ for some $r>1$. This follows from the fact that the simple random walk is absorbed by $B$ almost surely, since the graph $\hat{C}$ is finite.

Let $v \in \theta C$; then we define the rational functions $d$ and $f$ by

$$
d(z)=\sum_{w \in \theta C \backslash\{v\}}\left(Q_{\theta C \backslash\{v\}}^{*}(z)\right)_{v, w} \quad \text { and } \quad f(z)=\left(Q_{\theta C \backslash\{v\}}^{*}(z)\right)_{v, v} .
$$

The functions $d$ and $f$ are independent of the choice of $v$, because $\operatorname{Aut}(\hat{C})$ acts transitively on $\theta C$. Furthermore, double transitivity implies

$$
d(z)=(\theta-1) \cdot\left(Q_{\theta C \backslash\{v\}}^{*}(z)\right)_{v, w}
$$

for any vertex $w \in \theta C \backslash\{v\}$. Now $d$ is the generating function of the probabilities that the simple random walk on $\hat{C}$ starting in some vertex $v$ in $\theta C$ hits a vertex in $\theta C \backslash\{v\}$ for the first time after exactly $n$ steps, whereas $f$ is the generating function of the probabilities that the random walk starting at $v$ returns to $v$ after $n$ steps without hitting a vertex in $\theta C \backslash\{v\}$ before. The start is counted as the first visit; thus $f(0)=1$. We call $d$ the transition function and $f$ the return function of $X$.

We write $G(z)$ instead of $G(o, o \mid z)$ if $X$ is a self-similar graph with origin vertex $o$.

Lemma 3. Let $X$ be a symmetrically self-similar graph with origin vertex o. Then

$$
G(z)=f(z) \cdot G(d(z))
$$

for all $z \in U(0,1)$.

This functional equation was shown for the Sierpiński graph in [24, 25] and [12]. It is a special case of Lemma 6 in [18].

The basic idea behind this identity is the substitution $z \mapsto d(z)$, which corresponds to a combinatorial path substitution reflecting the self-similarity of the graph. For more details see Section 4 in 18]. The number of orbits of $\operatorname{Aut}(\hat{C})$ on the set $\{(v, w) \mid v, w \in \theta C\}$ is the number of 'types' of transitions from one boundary vertex in $\theta C$ to another. It is the number of variables needed for the functional equations of the Green functions. Similarly, this is the number of variables in the renormalization equations for constructing Brownian motion on fractals (see, for example, [20]). Axiom (S2) ensures that only one variable is needed. Our techniques would apply to more general self-similar graphs if the dynamics of multidimensional transition functions could be understood.

Let $\mathcal{D}_{0} \subset \mathbb{C} \cup\{\infty\}$ be the set of all poles of the function $f$, and let $\mathcal{D}$ be the set of all $z \in \mathbb{C} \cup\{\infty\}$ such that $d^{n}(z) \in \mathcal{D}_{0}$ for some $n \in \mathbb{N}_{0}$. The following lemma corresponds to Lemmata 9, 11, 12 and Theorem 4 in [18].

Lemma 4. Let $X$ be a symmetrically self-similar graph with bounded geometry.

(a) The point $z=1$ is a repelling fixed point of the transition function $d$.

(b) The point $z=0$ is a super-attracting fixed point of $d$, and it is the only attracting fixed point.

(c) The Fatou set $\mathcal{F}$ of $d$ is the immediate basin of attraction of $z=0$.

(d) The Julia set $\mathcal{J}$ of $d$ is a subset of $(\mathbb{R} \cup\{\infty\}) \backslash(-1,1)$.

(e) The set $\mathcal{D}$ is a subset of $(\mathbb{R} \cup\{\infty\}) \backslash(-1,1)$. 
(f) Every Green function $G(x, y \mid \cdot)$ is holomorphic in $\mathcal{F} \backslash \mathcal{D}$ and cannot be continued holomorphically to any point in $\mathcal{J}$.

In [18] this information was used for the description of the spectrum of the Laplacian. Here it is the basic tool for studying the transition probabilities.

We recall that a point $z_{0}$ is called an exceptional point of the map $d$ if the smallest completely invariant set containing $z_{0}$ is finite. Let $z_{0} \in \mathcal{F}$ be an exceptional point of $d$. Then the smallest completely invariant set containing $z_{0}$ must also contain 0 , since $\mathcal{F}$ is the immediate basin of attraction of 0 . Since a rational function of degree greater or equal to two has at most two exceptional points, we have $d\left(z_{0}\right)=0$ and $d(0)=z_{0}$ (see the proof of Theorem 4.1.2 in Beardon's book [4]). Thus $d$ has at most the exceptional point $z=0$. Since $0 \notin \mathcal{D}_{0}$, the set of accumulation points of $\mathcal{D}$ coincides with $\mathcal{J}$ (see [4, Theorems 4.2.7 and 4.2.8]). Hence $\overline{\mathcal{D}}=\mathcal{D} \cup \mathcal{J}$ and $\mathcal{F} \backslash \mathcal{D}=(\mathbb{C} \cup\{\infty\}) \backslash \overline{\mathcal{D}}$. We remark that either $z=0$ is exceptional and $d$ is conjugated to a polynomial, or there are no exceptional points at all. Actually, both cases can occur, as we will show by examples in Sections 8 and 9 .

Corollary 1. Let $X$ be a symmetrically self-similar graph with bounded geometry and origin vertex $o$. The Green function $G$ has a unique holomorphic continuation to $\mathcal{F} \backslash \mathcal{D}$, and

$$
G(z)=\prod_{k=0}^{\infty} f\left(d^{k}(z)\right)
$$

for all $z \in \mathcal{F} \backslash \mathcal{D}$. This convergence is uniform on any compact subset of $\mathcal{F} \backslash \mathcal{D}$. If $X$ is not bipartite, then $\mathcal{F} \backslash \mathcal{D}$ contains an interval $(-r,-1]$ for some $r>1$.

Proof. The first part is a consequence of Lemma 4 Let $X$ be not bipartite. Then, by Lemma 2, the cell graph $\hat{C}$ is not bipartite. Hence $d$ is neither even nor odd. Therefore $|d(-1)|<1$, and $\mathcal{F} \backslash \mathcal{D}$ contains $(-r,-1]$ for some $r>1$.

\section{Geometric And Probabilistic dimensions of SELF-Similar GRAPhS}

Let $X$ be a symmetrically self-similar graph with respect to $F$ and $\psi$. We call

$$
\tau=d^{\prime}(1)
$$

the time scaling factor of $X$. Then $\tau$ is the expected number of steps of the simple random walk on the cell graph $\hat{C}$ for hitting $\theta C \backslash\{v\}$, when starting at some $v \in \theta C$. Let $C_{2}$ be any 2-cell of $X$. Then the number of 1-cells in a 2-cell

$$
\mu=\left|\left\{C \in \mathcal{C}(F) \mid C \subset V \hat{C}_{2}\right\}\right|
$$

is called the mass scaling factor of $X$. Axiom (S1) implies that $\mu$ is independent of the choice of the 2-cell $C_{2}$. Furthermore, we call

$$
d_{s}=\frac{2 \log (\mu)}{\log (\tau)}
$$

the spectral dimension of $X$. The spectral dimension was first introduced in physics literature using the density of states of the Laplacian; see [1, 25] and the references therein. Later on, the exponent $d_{s}$ was studied from several points of view; see [2, 13, 9, 12, 14, 16, 17]. 
A measure $m$ on the set of vertices $V Y$ of a countable graph $Y$ is invariant with respect to a transition matrix $P=(p(x, y))_{x, y \in V Y}$ if $m \cdot P=m$, where $m$ is considered as a row vector. Written in coordinates, this is

$$
m(x)=\sum_{y \in V Y} m(y) p(y, x)
$$

for any $x \in V Y$. If $Y$ is connected and finite, then the unique invariant probability measure of the simple random walk is given by

$$
m(x)=\frac{\operatorname{deg}_{Y}(x)}{\sum_{x \in V Y} \operatorname{deg}_{Y}(x)}=\frac{\operatorname{deg}_{Y}(x)}{2|E Y|} .
$$

Lemma 5. Let $X$ be a symmetrically self-similar graph, and let $C$ be any cell of $X$. Then $m: V \hat{C} \rightarrow \mathbb{R}$, defined by

$$
m(x)=\frac{\beta(x)}{\mu \theta},
$$

is the unique invariant probability measure of the simple random walk on $\hat{C}$.

Proof. Let $x$ be any vertex in $V \hat{C}$. Since the cell graph $\hat{C}$ consists of $\mu$ amalgamated $\theta$-complete graphs, we have $\operatorname{deg}_{\hat{C}}(x)=(\theta-1) \beta(x)$ and $2|E \hat{C}|=\mu \theta(\theta-1)$. This implies the lemma.

In the following we will make use of path arguments. For similar path decompositions, see [11, [12, and especially [18]. These constructions can be seen in the context of more general generating functions; see Goulden and Jackson [10].

Let $C$ be a cell of a symmetrically self-similar graph $X$. For $v, w \in \theta C$ and $B \subset \theta C$ let $\Pi_{B}(v, w)$ be the set of paths from $v$ to $w$ which do not hit any vertex in $B$ except for their start and their end vertex. The set of paths in $\Pi_{B}(v, w)$ with positive length is denoted by $\Pi_{B}^{*}(v, w)$.

The weight of a path $\pi=\left(x_{0}, \ldots, x_{n}\right)$ in $\hat{C}$ is defined by

$$
W(\pi \mid z)=\prod_{i=0}^{n-1} \frac{z}{\operatorname{deg}_{\hat{C}}\left(x_{i}\right)}=\frac{z^{n}}{(\theta-1)^{n}} \prod_{i=0}^{n-1} \frac{1}{\beta\left(x_{i}\right)}
$$

for $z \in \mathbb{C}$. Now $W(\pi \mid 1)$ is the probability, that the simple random walk on $\hat{C}$, starting at $x_{0}$, follows the path $\pi$ in its first $n$ steps. For a set of paths $\Pi$ we set

$$
W(\Pi \mid z)=\sum_{\pi \in \Pi} W(\pi \mid z) .
$$

For $\Pi_{1} \subset \Pi\left(x_{1}, x_{2}\right)$ and $\Pi_{2} \subset \Pi\left(x_{2}, x_{3}\right)$ we write $\Pi_{1} \circ \Pi_{2}$ for the set of all concatenations of paths in $\Pi_{1}$ with paths in $\Pi_{2}$. Then

$$
W\left(\Pi_{1} \circ \Pi_{2} \mid z\right)=W\left(\Pi_{1} \mid z\right) W\left(\Pi_{2} \mid z\right) .
$$

Let $v$ again be a vertex in $\theta C$. We define $\hat{u}: U(0,1) \rightarrow \mathbb{C}$ and $u: U(0,1) \rightarrow \mathbb{C}$ by

$$
\hat{u}(z)=W\left(\Pi_{\{v\}}^{*}(v, v) \mid z\right) \quad \text { and } \quad u(z)=W\left(\Pi_{\theta C}^{*}(v, v) \mid z\right) .
$$

Then $\hat{u}$ is the generating function of the probabilities that the simple random walk on $\hat{C}$, starting at $v$, returns for the first time to $v$ after exactly $n$ steps, where $n>0$, and $u$ is the generating function of the first return after $n$ steps without hitting any vertex in $\theta C \backslash\{v\}$ before. Since $\operatorname{Aut}(\hat{C})$ acts transitively on $\theta C$, the functions $\hat{u}$ and $u$ are independent of the choice of $v$. 
Lemma 6. Let $C$ be a cell of a symmetrically self-similar graph $X$, and let $v$ and $w$ be two different vertices in $\theta C$. Then

$$
f(z)=W\left(\Pi_{\theta C \backslash\{v\}}(v, v) \mid z\right)=1+W\left(\Pi_{\theta C \backslash\{v\}}^{*}(v, v) \mid z\right)=\frac{1}{1-u(z)}
$$

and

$$
d(z)=(\theta-1) W\left(\Pi_{\theta C \backslash\{v\}}(v, w) \mid z\right)=(\theta-1) f(z) W\left(\Pi_{\theta C}(v, w) \mid z\right) .
$$

Proof. Since $\Pi_{\theta C \backslash\{v\}}(v, v)$ is the set of all paths in $\hat{C}$ from $v$ to $v$ which do not hit vertices in $\theta C \backslash\{v\}$, we get

$$
f(z)=W\left(\Pi_{\theta C \backslash\{v\}}(v, v) \mid z\right)=1+W\left(\Pi_{\theta C \backslash\{v\}}^{*}(v, v) \mid z\right) .
$$

Every path in $\Pi_{\theta C \backslash\{v\}}^{*}(v, v)$ can be obtained by concatenating a finite number of paths in $\Pi_{\theta C}^{*}(v, v)$; therefore

$$
f(z)=1+\sum_{k=1}^{\infty} u(z)^{k}=\frac{1}{1-u(z)}
$$

The definition of $d$ implies

$$
d(z)=\sum_{w \in \theta C \backslash\{v\}} W\left(\Pi_{\theta C \backslash\{v\}}(v, w) \mid z\right)=f(z) \sum_{w \in \theta C \backslash\{v\}} W\left(\Pi_{\theta C}(v, w) \mid z\right) .
$$

Here we used the fact, that

$$
\left.\Pi_{\theta C \backslash\{v\}}(v, w)=\Pi_{\theta C \backslash\{v\}}(v, v) \circ \Pi_{\theta C}(v, w)\right) .
$$

Axiom (S2) now yields the rest of the statement.

Lemma 7. Let $X$ be a symmetrically self-similar graph. Then

$$
\hat{u}(z)=u(z)+\frac{d(z)}{f(z)} \cdot \frac{1}{1-\frac{\theta-2}{\theta-1} d(z)} \cdot \frac{d(z)}{\theta-1} .
$$

Proof. Let $C$ be any cell of $X$ and let $v$ be any vertex in $\theta C$. If we denote by $\Pi(v)$ those paths in $\Pi_{\{v\}}^{*}(v, v)$, which hit a vertex in $\theta C \backslash\{v\}$, then

$$
\Pi_{\{v\}}^{*}(v, v)=\Pi_{\theta C}^{*}(v, v) \uplus \Pi(v),
$$

and therefore $\hat{u}(z)=u(z)+W(\Pi(v) \mid z)$. For an integer $n$ in $\mathbb{N}_{0}$ let $\Lambda_{n}(v)$ be the set of all $(n+1)$-tuples $a=\left(a_{0}, \ldots, a_{n}\right)$ in $(\theta C \backslash\{v\})^{n+1}$ such that $a_{k} \neq a_{k+1}$ for $0 \leq k<n$. If we write $\Pi_{a}(v)$ for the set

$$
\Pi_{\theta C \backslash\left\{a_{0}\right\}}\left(a_{0}, a_{1}\right) \circ \cdots \circ \Pi_{\theta C \backslash\left\{a_{n-1}\right\}}\left(a_{n-1}, a_{n}\right),
$$

then, by Lemma 6

$$
W\left(\Pi_{a}(v) \mid z\right)=\frac{d(z)^{n}}{(\theta-1)^{n}} .
$$

Now we can decompose $\Pi(v)$ in the following way:

$$
\Pi(v)=\biguplus_{n \in \mathbb{N}_{0}} \biguplus_{a \in \Lambda_{n}(v)} \Pi_{\theta C}\left(v, a_{0}\right) \circ \Pi_{a}(v) \circ \Pi_{\theta C \backslash\left\{a_{n}\right\}}\left(a_{n}, v\right) .
$$


From this we obtain

$$
\begin{aligned}
W(\Pi(v) \mid z) & =\sum_{n \in \mathbb{N}_{0}} \sum_{a \in \Lambda_{n}(v)} W\left(\Pi_{\theta C}\left(v, a_{0}\right) \mid z\right) W\left(\Pi_{a}(v) \mid z\right) W\left(\Pi_{\theta C \backslash\left\{a_{n}\right\}}\left(a_{n}, v\right) \mid z\right) \\
& =\sum_{n \in \mathbb{N}_{0}} \sum_{a \in \Lambda_{n}(v)} \frac{d(z)}{(\theta-1) f(x)} \cdot \frac{d(z)^{n}}{(\theta-1)^{n}} \cdot \frac{d(z)}{\theta-1} \\
& =\frac{d(z)}{f(z)} \cdot \frac{1}{1-\frac{\theta-2}{\theta-1} d(z)} \cdot \frac{d(z)}{\theta-1}
\end{aligned}
$$

using Lemma 6 and the fact that $\left|\Lambda_{n}(v)\right|=(\theta-1)(\theta-2)^{n}$.

For the following statements we recall that $\beta=1$ if and only if the graph has bounded geometry, see Theorem 2, Let the simple random walk on $\hat{C}$ start at a vertex $v$ in $\theta C$. Then $f(1)$ is the expected number of returns to $v$ (the start is counted as the first visit) before hitting another vertex in $\theta C$. The resistance scaling factor is defined by the relation $\tau=\mu \rho$; see [2, equation (6.29)].

Theorem 4. Let $X$ be a symmetrically self-similar graph. Then $f(1)=\beta \rho$.

Proof. Since $\hat{u}(1)=1$ and $d(1)=1$, we obtain

$$
\hat{u}^{\prime}(1)=\lim _{z \rightarrow 1} \frac{\hat{u}(z)-1}{z-1}=\lim _{z \rightarrow 1} \frac{d(z)+(\theta-1)}{f(z)((\theta-1)-(\theta-2) d(z))} \cdot \frac{d(z)-1}{z-1}=\frac{\theta \tau}{f(1)},
$$

using Lemmata 6 and 7 . Now $\hat{u}^{\prime}(1)$ is the expected number of steps of the first return of a simple random walk on $\hat{C}$ starting at a vertex $v$ in $\theta C$. Thus $\hat{u}^{\prime}(1) m(v)=1$. A proof of this well known identity can be found in the book of Brémaud; see [6] Theorem 3.2]. By Lemma [5 we have

$$
\hat{u}^{\prime}(1)=\frac{1}{m(v)}=\frac{\mu \theta}{\beta},
$$

completing the proof.

Corollary 2. Let $X$ be a symmetrically self-similar graph. Then $\tau \beta>\mu$.

If $X$ is a symmetrically self-similar graph with bounded geometry, then the last corollary implies $0<d_{s}<2$.

\section{Asymptotic AnALYsis}

Throughout the rest of this paper let $X$ be a symmetrically self-similar graph with bounded geometry and origin vertex $o$. We recall that symmetrically selfsimilar graphs with an origin vertex either have bounded geometry, or the origin vertex is the only vertex with infinite degree; see Theorem 3

Since $d(1)=1$ and $\tau=d^{\prime}(1)>2$, there exists a holomorphic local inverse $d^{-1}$ of $d$ in a neighbourhood of $z=1$ which has a attracting fixed point at $z=1$. For $n \in \mathbb{N}$ we write $d^{-n}$ for the $n$-fold iterate of $d^{-1}$. Let $z_{0} \in \mathbb{C}, r>0$ and $0<\delta \leq \pi$; then we write $U_{\delta}\left(z_{0}, r\right)$ for the set of all $z \in \mathbb{C} \backslash\left\{z_{0}\right\}$ such that $\left|z-z_{0}\right|<r$ and $\left|\arg \left(z_{0}-z\right)\right|<\delta$. In particular, we have $U_{\pi}(1, r)=U(1, r) \backslash[1,1+r)$. Furthermore, we write $S(b)$ for the horizontal strip

$$
S(b)=\left\{z \in \mathbb{C}|| \operatorname{Im}(z) \mid<\frac{\pi}{\log (b)}\right\} .
$$


Lemma 8. Let $H: U_{\pi}(1, R) \rightarrow \mathbb{C}$ be a holomorphic function for some $R>0$ which satisfies the equation $H(z)=H(d(z))$ whenever $z \in U_{\pi}(1, R)$ and $d(z) \in U_{\pi}(1, R)$. Then there exist a 1-periodic, holomorphic function $\omega$ defined on the strip $S(\tau)$ and an $r \in(0, R)$ such that

$$
H(z)=\omega\left(\frac{\log (1-z)}{\log (\tau)}\right)+\mathcal{O}_{\delta}(|z-1|)
$$

for $z \in U_{\delta}(1, r)$ for all $0<\delta<\pi$. The function $\omega$ is constant if and only if $H$ is constant.

This lemma was proved by one of the authors in [27, Lemma 5]. The proof of the lemma and the following theorem are based on ideas of de Bruijn [7], Odlyzko [23] and Grabner and Woess [12]. A detailed discussion of the methods can be found in the book [28, Section 16].

The point $z=1$ is a non-polar singularity of any Green function of any recurrent random walk on an infinite, locally finite graph; see for example [18, Lemma 10]. Lemma 3 in [18] implies that the simple random walk on any symmetrically selfsimilar graph with bounded geometry is recurrent.

Theorem 5. Let $X$ be a symmetrically self-similar graph with bounded geometry and origin vertex $o$. Then there exists a 1-periodic, holomorphic function $\omega$ on the strip $S(\tau)$, such that the Green function $G$ has the local singular expansion

$$
G(z)=(1-z)^{\frac{d_{s}}{2}-1}\left(\omega\left(\frac{\log (1-z)}{\log (\tau)}\right)+\mathcal{O}_{\delta}(|z-1|)\right)
$$

for $z \in U_{\delta}(1, r)$ and $0<\delta<\pi$, where $r>0$.

Proof. We substitute $G(z)=(1-z)^{\alpha} H^{+}(z)$ into (1), where $H^{+}$is holomorphic in $U_{\pi}(1,1)$ and $\alpha \in \mathbb{R}$ is some constant. This yields $H^{+}(z)=f(z) q(z)^{\alpha} H^{+}(d(z))$, where $q$ is given by

$$
q(z)=\frac{1-d(z)}{1-z}
$$

and $q(1)=\tau$. Furthermore, we define

$$
H^{-}(z)=\prod_{n=-\infty}^{-1} f\left(d^{n}(z)\right) q\left(d^{n}(z)\right)^{\alpha} .
$$

Since $f$ and $q$ are holomorphic in $U(1, R)$ for some $R>0$, the last product converges if $f(1) q(1)^{\alpha}=1$. Hence, we have to choose

$$
\alpha=\frac{\log (\mu)}{\log (\tau)}-1=\frac{d_{s}}{2}-1 .
$$

Moreover, the convergence is uniform in $U(1, R)$, and therefore the function $H^{-}$ is holomorphic in $U(1, R)$. Obviously, $H^{-}(d(z))=H^{-}(z) f(z) q(z)^{\alpha}$ for all $z \in$ $U(1, R)$ such that $d(z) \in U(1, R)$. Let $H: U_{\pi}(1, R) \rightarrow \mathbb{C}$ be defined by $H(z)=$ $H^{-}(z) H^{+}(z)$; then $H$ is holomorphic and satisfies

$$
\begin{aligned}
H(z) & =H^{-}(z) H^{+}(z)=H^{-}(z) f(z) q(z)^{\alpha} H^{+}(d(z)) \\
& =H^{-}(d(z)) H^{+}(d(z))=H(d(z))
\end{aligned}
$$


whenever $z$ and $d(z)$ are contained in $U_{\pi}(1, R)$. Applying Lemma 8 to $H$, we get a 1-periodic holomorphic function $\omega$ on the strip $S(\tau)$ and an $r \in(0, R)$ such that

$$
H(z)=\omega\left(\frac{\log (1-z)}{\log (\tau)}\right)+\mathcal{O}_{\delta}(|z-1|)
$$

for $z \in U_{\delta}(1, r)$, for all $0<\delta<\pi$. Since $H^{-}(1)=1$ and $H^{-}$is holomorphic in $U(1, r)$, we finally obtain

$$
G(z)=(1-z)^{\alpha} \frac{H(z)}{H^{-}(z)}=(1-z)^{\alpha}\left(\omega\left(\frac{\log (1-z)}{\log (\tau)}\right)+\mathcal{O}_{\delta}(|z-1|)\right)
$$

for $z \in U_{\delta}(1, r)$ and $0<\delta<\pi$.

We will now use the method of 'singularity analysis' due to Flajolet and Odlyzko [8], in order to obtain the asymptotic behaviour of $p^{(n)}(o, o)$. It should be mentioned here that a real Tauberian theorem would not reveal the oscillating nature.

Theorem 6. Let $X$ be a symmetrically self-similar graph with bounded geometry and origin vertex $o$. If $X$ is not bipartite, then

$$
p^{(n)}(o, o)=n^{-\frac{d_{s}}{2}}\left(\sigma\left(\frac{\log (n)}{\log (\tau)}\right)+\mathcal{O}\left(n^{-1}\right)\right),
$$

where $\sigma: S\left(\tau^{2}\right) \rightarrow \mathbb{C}$ is a 1-periodic, holomorphic function given by its Fourier series

$$
\sigma(z)=\sum_{k=-\infty}^{\infty} \frac{\hat{\omega}(-k)}{\Gamma\left(1-\frac{\log (\mu)+2 k \pi i}{\log (\tau)}\right)} \cdot e^{2 k \pi i z} .
$$

If $X$ is bipartite, then $p^{(2 n+1)}(o, o)=0$ and

$$
p^{(2 n)}(o, o)=2(2 n)^{-\frac{d_{s}}{2}}\left(\sigma\left(\frac{\log (2 n)}{\log (\tau)}\right)+\mathcal{O}\left(n^{-1}\right)\right) .
$$

Proof. Since the 1-periodic function $\omega$ of Theorem [5 is holomorphic in $S(\tau)$, we have

$$
\hat{\omega}(k)=\mathcal{O}_{\varepsilon}\left(e^{-2 \pi|k|\left(\frac{\pi}{\log (\tau)}-\varepsilon\right)}\right)
$$

for all $k \in \mathbb{Z}$ and $\varepsilon>0$, where $\hat{\omega}(k)$ denotes the $k$-th Fourier coefficient of $\omega$. Therefore it is possible to apply the method of 'singularity analysis'. For the technical details of the transfer we refer to [23, Section 5] and [28, Section 16].

If $X$ is not bipartite, then $z=1$ is the only singularity of $G$ at the boundary of the unit disk. The asymptotic of $p^{(n)}(o, o)$ is now a consequence of the singularity analysis.

If $X$ is bipartite, then $G$ is an even function. Hence $p^{(2 n+1)}(o, o)=0$. Furthermore, $G$ has the same singularity at $z=-1$ as at $z=1$. Thus we have to add up the terms corresponding to the singularities $z=1$ and $z=-1$ for the asymptotics of $p^{(2 n)}(o, o)$.

We remark here that the function $H$ of the proof of Theorem 5 maps the interval $(1-r, 1)$ to $\mathbb{R}$. By inspection of the proof of Lemma 8 in $[27$, we see that both $\omega$ and $\sigma$ map $\mathbb{R}$ to $\mathbb{R}$. Hence the Fourier coefficients of $\omega$ and $\sigma$ satisfy

$$
\hat{\omega}(-k)=\overline{\hat{\omega}(k)} \quad \text { and } \quad \hat{\sigma}(-k)=\overline{\hat{\sigma}(k)}
$$

for all $k \in \mathbb{Z}$. It is possible to compute the Fourier coefficients of $\omega$ for a given graph $X$ numerically; see [27]. 


\section{Oscillation}

Let $X$ be a symmetrically self-similar graph with bounded geometry and origin vertex $o$. We have shown that the asymptotics of $p^{(n)}(o, o)$ carries an oscillating factor given by

$$
\sigma\left(\frac{\log (n)}{\log (\tau)}\right)
$$

where $\sigma$ is a 1-periodic, holomorphic function given by the Fourier series in Theorem [6 In this section we will give a necessary condition for the case that the 1-periodic function $\sigma$ is constant. First of all we note that the following statements are equivalent:

- The 1-periodic function $\sigma$ is constant.

- The 1-periodic function $\omega$ of Theorem 5 is constant.

- The function $H$ given in the proof of Theorem 5 is constant.

We denote by $[a, b]$ a closed interval in $\mathbb{R} \cup\{\infty\}$, where $a, b \in \mathbb{R} \cup\{\infty\}$. Here $a$ and $b$ do not necessarily satisfy the inequality $a \leq b$. For example, $[1,-2]$ is the set $\{z \in \mathbb{R} \mid z \geq 1\} \cup\{\infty\} \cup\{z \in \mathbb{R} \mid z \leq-2\}$. The same notation is used for open and for half open intervals.

Theorem 7. If $\sigma$ is constant, then the Julia set $\mathcal{J}$ of the transition function $d$ is a closed interval $[1, a]$ in the extended real line $\mathbb{R} \cup\{\infty\}$, where $a \in(1,-1]$.

Proof. The Julia set $\mathcal{J} \subset \mathbb{R} \cup\{\infty\}$ is either a Cantor set or a closed interval; see [4, Theorem 5.7.1]. We will use the notation of the proof of Theorem 5. If $\sigma$ is constant, then the function $H$ is also constant. Hence we obtain

$$
G(z)=\frac{C(1-z)^{\alpha}}{H^{-}(z)}
$$

for $z \in U(1, r)$, where $C$ is some constant and $r>0$. Since $z=1$ is not a polar singularity by Lemma 10 in $[18$, we have $\alpha \notin \mathbb{Z}$. Therefore $G$ has no analytic continuation to $[1,1+r)$, and Corollary 1 implies that $[1,1+r) \subset \mathcal{J} \cup \mathcal{D}$. Furthermore, the set of accumulation points of $\mathcal{D}$ coincide with $\mathcal{J}$. Thus $\mathcal{J}$ contains the interval $[1,1+r)$ and must therefore be itself a closed interval in $\mathbb{R} \cup\{\infty\}$.

Corollary 3. If $\mathcal{J}$ is a Cantor set, then $\sigma$ is non-constant.

It is not clear whether the opposite direction of this corollary is also true. For rational functions with real Julia set there is a general method to decide whether their Julia set is an interval or a Cantor set. For this purpose we refer to Inninger's detailed discussion of rational functions in Sections 3 and 4 of [13]. However, for a given transition function $d$ the following criterion may be useful as well: If we can find a real number $z_{0}>1$ which is smaller than some $d$-backwards iterate of $z=1$ together with an integer $n$ such that $\left|d^{n}\left(z_{0}\right)\right|<1$, then $\mathcal{J}$ is a Cantor set.

\section{TRANSITION PROBABILITIES BETWEEN ARBITRARY VERTICES}

Let $X$ be a symmetrically self-similar graph with bounded geometry and let $P$ be the matrix of transition probabilities for the simple random walk on $X$.

Lemma 9. If $X$ is not bipartite, then $P$ is strongly aperiodic, that is, there exists a number $n_{0}$ such that $\inf \left\{p^{(n)}(x, x) \mid x \in X\right\}>0$ for all $n \geq n_{0}$. In general, $P^{2}$ is strongly aperiodic. 
Proof. Since $X$ has bounded geometry and is connected, there exists a $c_{2}>0$ such that $p^{(2)}(x, x) \geq c_{2}$ for all $x \in V X$. Thus $P^{2}$ is strongly aperiodic.

If $X$ is not bipartite, then the cell graph $\hat{C}$ of $X$ is not bipartite by Lemma 2 Hence for any vertex $v \in V \hat{C}$ there exists a closed path in $\hat{C}$ from $v$ to $v$ of odd length. By adding a path of the form $(v, w, v)$, where $w$ is adjacent to $v$ in $\hat{C}$, we can extend such a path for $v$ by an even number of edges. Thus there is an odd number $\ell$ such that for any $x \in V \hat{C}$ we can find a closed path from $v$ to $v$ of length $\ell$. Since any vertex $x \in V X$ is contained in at least one cell graph, there is a real number $c_{1}>0$ such that $p^{(\ell)}(x, x) \geq c_{1}$ for all $x \in V X$. As every $n \geq \ell$ can be written in the form $r \cdot 2+s \cdot \ell$ with $r, s \in \mathbb{N}_{0}$, strong aperiodicity follows.

We remark here that it is possible to choose $\ell=3$ if $\theta \geq 3$, since the cell graph consists of $\mu$ copies of the $\theta$-complete graph.

Theorem 8. Let $x$ and $y$ be two vertices of a symmetrically self-similar graph $X$ with bounded geometry and origin vertex o. If $X$ is not bipartite, then

$$
p^{(n)}(x, y)=n^{-\frac{d_{s}}{2}}\left(\sigma\left(\frac{\log (n)}{\log (\tau)}\right)+o(1)\right) .
$$

If $X$ is bipartite, let $r$ be an element of $\{0,1\}$ such that $d(x, y) \equiv r \bmod 2$. Then $p^{(2 n+1-r)}(x, y)=0$ and

$$
p^{(2 n+r)}(x, y)=2(2 n+r)^{-\frac{d_{s}}{2}}\left(\sigma\left(\frac{\log (2 n+r)}{\log (\tau)}\right)+o(1)\right) .
$$

Proof. If $X$ is not bipartite, then

$$
\lim _{n \rightarrow \infty} \frac{p^{(n)}(x, y)}{p^{(n)}(y, y)}=1,
$$

by [12, Theorem 2], since $P$ is irreducible, strongly aperiodic and recurrent. Thus

$$
\frac{p^{(n)}(x, y)}{p^{(n)}(o, o)}=\frac{p^{(n)}(x, y)}{p^{(n)}(y, y)} \cdot \frac{p^{(n)}(y, y)}{p^{(n)}(y, o)} \cdot \frac{p^{(n)}(y, o)}{p^{(n)}(o, o)}=1+o(1) .
$$

Now Theorem 6 implies the statement.

If $X$ is bipartite, then we can partition $V X$ into classes $V_{1}$ and $V_{2}$ such that edges in $E X$ only connect vertices in $V_{1}$ with vertices in $V_{2}$. Then $P^{2}$ restricted to $V_{i}$ for $i \in\{1,2\}$ is irreducible, strongly aperiodic and recurrent. If $r=0$, then $x$ and $y$ are in the same class. Hence $p^{(2 n+1)}(x, y)=0$ and

$$
\frac{p^{(2 n)}(x, y)}{p^{(2 n)}(o, o)}=1+o(1)
$$

If $r=1$, then $p^{(2 n)}(x, y)=0$ and

$$
\frac{p^{(2 n+1)}(x, y)}{p^{(2 n)}(o, o)}=\sum_{v \in V X} \frac{p(x, v) p^{(2 n)}(v, x)}{p^{(2 n)}(o, o)}=\sum_{v \in V X} p(x, v)(1+o(1))=1+o(1) .
$$

Note that the above sum is finite, since there are only finitely many vertices $v \in V X$ such that $p(x, v)>0$. Furthermore,

$$
(2 n)^{-\frac{d_{s}}{2}}=(2 n+1)^{-\frac{d_{s}}{2}}(1+o(1)) .
$$


As $\sigma$ is a 1-periodic, holomorphic function, it is uniformly continuous on the real line. We have $\log (2 n+1)-\log (2 n)=O\left(n^{-1}\right)$, and therefore

$$
\sigma\left(\frac{\log (2 n)}{\log (\tau)}\right)=\sigma\left(\frac{\log (2 n+1)}{\log (\tau)}\right)+o(1)
$$

which completes the proof.

Jones computed estimates for the transition probabilities $p^{(n)}(x, y)$ on the Sierpiński graph which are uniform in space $(x, y \in V X)$ and time $(n \in \mathbb{N})$. His result yields the correct asymptotic type

$$
p^{(n)}(x, y) \asymp n^{-\frac{\log (3)}{\log (5)}}
$$

as $n \rightarrow \infty$; see [14.

\section{Geodesic transition probabilities and the line Graph}

Example 1. Let the cell graphs of a self-similar graph $X$ be isomorphic to the line $L_{n}$ of length $n \geq 2$ (Figure 1a). If the graph $X$ has an origin vertex $o$, then it consists of an arbitrary number of one-sided infinite lines which are amalgamated at their initial vertex $o$, see Figure 1 b. If there is no origin vertex, then $X$ is the two-sided infinite line. The corresponding transition function $d_{n}$ is conjugated to the $n$-th Chebychev polynomial with conjugacy map $z \rightarrow \frac{1}{z}$,

$$
d_{n}(z)=z^{n}\left(\sum_{k=0}^{\left\lfloor\frac{n}{2}\right\rfloor}\left(\begin{array}{c}
n \\
2 k
\end{array}\right)\left(1-z^{2}\right)^{k}\right)^{-1} \quad \text { and } \quad d_{n}(z)=T_{n}\left(\frac{1}{z}\right)^{-1} ;
$$

see [18 Example 1]. As $\mathcal{J}\left(T_{n}\right)=[-1,1]$, we get

$$
\mathcal{J}\left(d_{n}\right)=[1,-1]=(\mathbb{R} \cup\{\infty\}) \backslash(-1,1) .
$$

We have $\theta=2, \mu=n, \beta=1, \rho=n$ and $\tau=n^{2}$. Furthermore, if there is an origin vertex, we have

$$
G(z)=\frac{1}{\sqrt{1-z^{2}}}
$$

for $z \in \mathbb{C} \backslash \mathcal{J}\left(d_{n}\right)$. Hence the function $\sigma$ is constant.

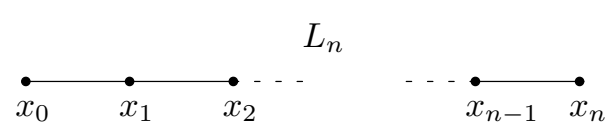

FiguRe 1,

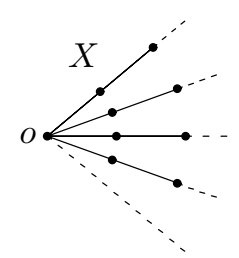

FiguRE $1 \mathrm{~b}$

To prove that lines as cell graphs are characterised by their return and transition functions, we state the following lemma.

Lemma 10. Let $Y$ be a locally finite, connected graph, and let $q^{(n)}(x, y)$ be the $n$-step transition probabilities of a reversible Markov chain on $Y$ which is of nearest neighbourhood type. Then $q^{(n)}(x, y) q^{(n)}(y, x) \leq 4^{1-n}$ for different vertices $x$ and $y$ 
which are at distance $n=d(x, y)$ from each other. Here equality holds if and only if

$$
\sum_{\substack{w \in V Y \\ d(y, w)<d(y, x)}} q(x, w)=\sum_{\substack{w \in V Y \\ d(x, w)<d(x, y)}} q(y, w)=1
$$

and

$$
\sum_{\substack{w \in V Y \\ d(x, w)<d(x, v)}} q(v, w)=\sum_{\substack{w \in V Y \\ d(y, w)<d(y, v)}} q(v, w)=\frac{1}{2}
$$

for all $v \in V Y \backslash\{x, y\}$; that is, the Markov chain can be projected to the simple random walk on the line $L_{n}$.

Proof. We prove a more general statement which is more adequate for induction: For a vertex $v \in V Y$ and a finite set of vertices $W$ of $V Y$ we write $d(v, W)=$ $\min \{d(v, w) \mid w \in W\}$. Let $n \in \mathbb{N}$, and let $A$ and $B$ be finite subsets of $V Y$ such that $d(a, B)=d(b, A)=n$ for all $a \in A$ and $b \in B$. For $k \in\{0, \ldots, n\}$ we introduce the level set $D_{k} \subset V Y$ by

$$
D_{k}=\{x \in V Y \mid d(x, A)=k, d(x, B)=n-k\} .
$$

These level sets are finite, $D_{0}=A$ and $D_{n}=B$. Furthermore, we define $D_{-1}=$ $D_{n+1}=\emptyset$, and

$$
m_{-}(x)=m(x) \sum_{y \in D_{k-1}} q(x, y), \quad m_{+}(x)=m(x) \sum_{y \in D_{k+1}} q(x, y),
$$

and $m_{0}(x)=m(x)-m_{-}(x)-m_{+}(x)$ for a vertex $x$ in $D_{k}$, where $k \in\{0, \ldots, n\}$. We define

$$
Q\left(D_{i}, D_{j}\right)=\sum_{v \in D_{i}} \sum_{w \in D_{j}} \frac{m(v) q^{(n)}(v, w)}{m_{+}(v)} \cdot \frac{m(w) q^{(n)}(w, v)}{m_{-}(w)}
$$

for $i, j \in\{0, \ldots, n\}$. Then we claim that $Q(A, B) \leq 4^{1-n}$. Equality holds if and only $m_{-}(x)=m_{+}(x)=\frac{1}{2} m(x)$ for any vertex $x$ in $D_{k}$, where $k \in\{1, \ldots, n-1\}$. $4^{1-n}$.

First of all we notice that the condition for equality indeed implies $Q(A, B)=$

We use induction over $n$. For $n=1$ the inequality is immediate. Now let $n>1$ and let the statement hold for all $k<n$. Factoring with respect to the $k$-th step and using the reversibility yields

$$
Q(A, B)=\sum_{a \in A} \sum_{b \in B} \frac{1}{m_{+}(a) m_{-}(b)}\left(\sum_{x \in D_{k}} m(x) q^{(k)}(x, a) q^{(n-k)}(x, b)\right)^{2}
$$

for $k \in\{1, \ldots, n-1\}$. Now we use the inequality $4 m_{-}(x) m_{+}(x) \leq m(x)^{2}$ for $x \in D_{k}$, where $k \in\{1, \ldots, n-1\}$. Here equality holds if and only if $m_{-}(x)=$ 
$m_{+}(x)=\frac{1}{2} m(x)$. Thus we obtain

$$
\begin{aligned}
& Q(A, B) \leq \frac{1}{4} \sum_{a \in A} \sum_{b \in B} \frac{1}{m_{+}(a) m_{-}(b)}\left(\sum_{x \in D_{k}} \frac{m(x) q^{(k)}(x, a)}{\sqrt{m_{-}(x)}} \cdot \frac{m(x) q^{(n-k)}(x, b)}{\sqrt{m_{+}(x)}}\right)^{2} \\
& \leq \frac{1}{4} \sum_{a \in A} \sum_{b \in B} \frac{1}{m_{+}(a) m_{-}(b)}\left(\sum_{x \in D_{k}} \frac{m(x)^{2} q^{(k)}(x, a)^{2}}{m_{-}(x)}\right) \\
& \times\left(\sum_{x \in D_{k}} \frac{m(x)^{2} q^{(n-k)}(x, b)^{2}}{m_{+}(x)}\right),
\end{aligned}
$$

using the Cauchy-Schwarz inequality for the second step. Now reversibility, together with the induction hypothesis for $Q\left(A, D_{k}\right)$ and $Q\left(D_{k}, B\right)$, implies that

$$
Q(A, B) \leq \frac{1}{4} Q\left(A, D_{k}\right) Q\left(D_{k}, B\right) \leq 4^{1-n} .
$$

As we can factor with respect to any $k \in\{1, \ldots, n-1\}$, we get the postulated conditions for equality.

Corollary 4. Let $d_{n}$ be the transition function and let $f_{n}$ be the return function of a cell graph which is isomorphic to the line $L_{n}$ of length $n \geq 2$. Then there is no other cell graph of a self-similar graph with bounded geometry with transition function $d_{n}$ or return function $f_{n}$.

Proof. Let the transition function of a cell graph be the function $d_{n}$. Then $d_{n}$ is either even or odd, whence the cell graph must be bipartite. By Lemma 2 the selfsimilar graph itself must be bipartite, and therefore $\theta=2$. As we have bounded geometry, $\beta=1$ by Theorem 2. The series expansion of $d_{n}$ in $z=0$ starts with $2^{1-n} z^{n}$. Now Lemma 10 implies that the cell graph $\hat{C}$ must be the line $L_{n}$.

Suppose $f_{n}$ is the return function of a cell graph $\hat{C}$ which is not isomorphic to $L_{n}$, and let $v$ be a vertex in the boundary $\theta C$. Since $f_{n}$ is even, we have $\theta=2$ and $\beta=1$ as before. There are vertices in $C$ which have edge degree strictly larger than 2 in the graph $\hat{C}$. Let $m$ be the minimal distance from $v$ to a vertex $x \in C$ with $\operatorname{deg}_{\hat{C}}(x) \geq 3$. Then the probability of returning to $v$ in exactly $2 m$ steps is strictly smaller than the corresponding probability for the line $L_{n}$. This means that the $2 m$-th coefficients of the series expansions of the return functions of $\hat{C}$ and $L_{n}$ around $z=0$ are different.

\section{Further EXAMPLES}

Well known examples of symmetrically self-similar graphs are the Viček graph and the Sierpiński graph. Both graphs do not belong to the following simple example of an infinite class of self-similar graphs.

Example 2. Let the cell graph $\hat{C}^{n}$ consist of $n$ copies of the $n$-complete graph which are amalgamated at one vertex $x$, where $n \geq 2$. The boundary of the cell contains exactly one vertex from each of these copies, but not the vertex $x$. Starting with these cell graphs, we can construct different symmetrically self-similar graphs in the sense of Theorem 1 The four vertices of the cell graph $\hat{C}^{4}$ in Figure $2 \mathrm{a}$ which constitute the boundary $\theta C^{4}$ of the cell are drawn bold. Figure $2 \mathrm{~b}$ shows the corresponding 4-cell graph $\hat{C}_{4}^{4}$. 


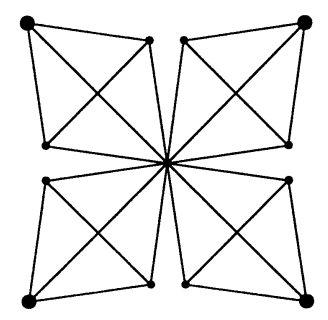

Figure 2a

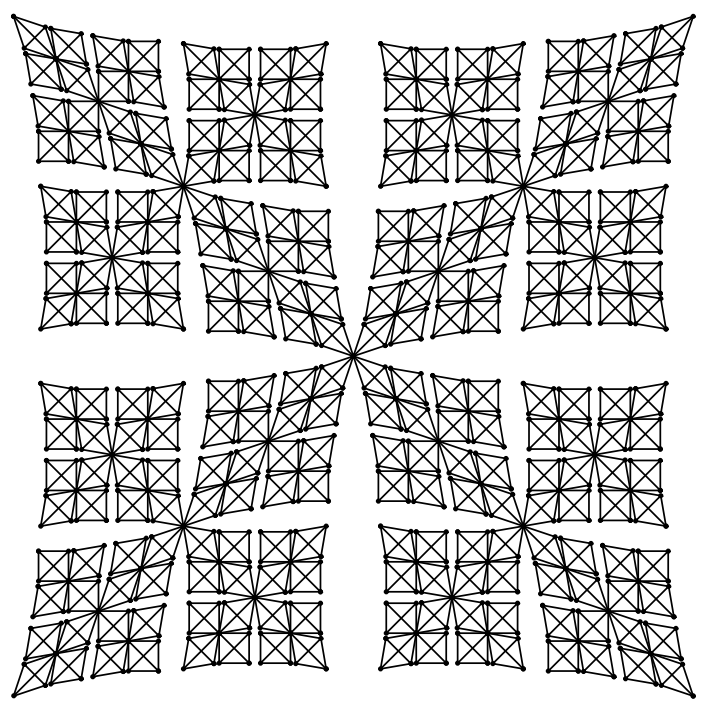

FiguRE $2 b$

The transition function $d_{n}$ of $\hat{C}^{n}$ is given by

$$
d_{n}(z)=\frac{z^{2}}{n(n-1)-2 n(n-2) z+\left(n^{2}-3 n+1\right) z^{2}}
$$

for $n \geq 2$. For the parameters we have $\mu=\theta=n, \beta=1, \tau=2 n$ and $\rho=2$. We remark that for $n=2$ the cells are isomorphic to the line $L_{2}, d_{2}(z)=T_{2}\left(\frac{1}{z}\right)^{-1}$, and $\mathcal{J}\left(d_{2}\right)=[1,-1]$; see Example 1, For $n \geq 3$ we have

$$
d\left(\frac{n-1}{n-3}\right)=1, \quad \text { but } \quad d^{2}\left(\frac{n-1}{n-2}\right)=\frac{n-1}{n(n-1)-1} \in(0,1) .
$$

Here $\frac{n-1}{n-3}$ has to be read as $\infty$ if $n=3$. As 1 and $\frac{n-1}{n-3}$ belong to $\mathcal{J}\left(d_{n}\right)$, whereas 0 and $\frac{n-1}{n-2}$ do not, the Julia set is not connected in $\mathbb{R} \cup\{\infty\}$. Hence $\mathcal{J}\left(d_{n}\right)$ is a Cantor set for $n \geq 3$. In the case that there is an origin vertex we can conclude that the 1-periodic function $\sigma$ is not constant, using Corollary 3 .

Many transition functions are of the form $d(z)=z^{n} / P(z)$, where $n$ is the minimal number of steps from one point in the boundary of a cell $C$ to another (in other words, $n=\operatorname{diam}(\theta C))$ and $P$ is a polynomial with integer coefficients.

As the following example shows, this is not true for all transition functions.

Example 3. The 3-dimensional, 4-scaled Sierpiński graph consists of 20 amalgamated 4-complete graphs. The 1-cell and 2-cell can be seen in Figure 3, Their boundary vertices are drawn fat. Each 4-complete graph is represented by a solid tetrahedron. Here we have

$$
d(z)=\frac{z^{4}\left(-486+9 z+23 z^{2}+2 z^{3}\right)}{-104976+227448 z-156168 z^{2}+31212 z^{3}+2958 z^{4}-887 z^{5}-41 z^{6}+2 z^{7}}
$$

and $\mu=20, \theta=4, \beta=1, \tau=\frac{4415}{113}$ and $\rho=\frac{883}{452}$. As

$$
d\left(\frac{9}{4}\right)=1 \quad \text { and } \quad d(2)=\frac{36}{53},
$$

the Julia set $\mathcal{J}$ is a Cantor set. If the constructed self-similar graph has an origin vertex, then the 1-periodic function $\sigma$ is not constant. 

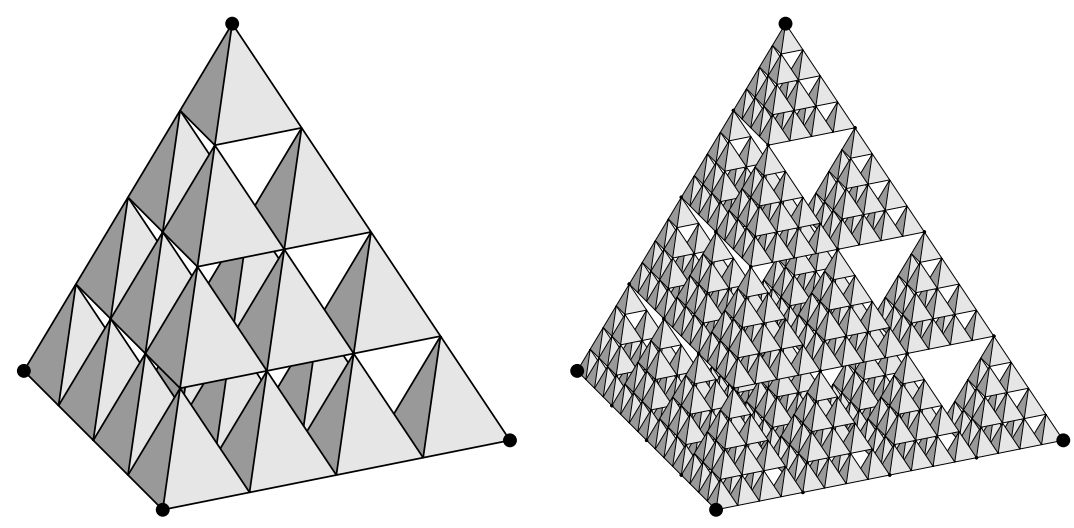

FiguRE 3.

\section{ACKNOWLEDGMENTS}

The authors want to thank Peter Grabner, Klaus Schmidt and Wolfgang Woess for fruitful discussions and financial support. Especially, the results in Section 4 would not have been possible without the contributions of Peter Grabner and Wolfgang Woess.

\section{REFERENCES}

1. S. Alexander and R. Orbach, Density of states on fractals: fractons, J. Physique Lettres 43 (1982), L625-L631.

2. M. T. Barlow, Diffusions on fractals, Lectures on probability theory and statistics (SaintFlour, 1995), Springer, Berlin, 1998, pp. 1-121. MR 2000a:60148

3. M. T. Barlow and E. A. Perkins, Brownian motion on the Sierpinski gasket, Probab. Theory Related Fields 79 (1988), no. 4, 543-623. MR 89g:60241

4. A. F. Beardon, Iteration of rational functions, Springer-Verlag, New York, 1991. MR 92j:30026

5. G. Ben Arous and T. Kumagai, Large deviations of Brownian motion on the Sierpinski gasket, Stochastic Process. Appl. 85 (2000), no. 2, 225-235. MR 2000k:60160

6. P. Brémaud, Markov chains, Springer-Verlag, New York, 1999. MR 2000k:60137

7. N. G. de Bruijn, An asymptotic problem on iterated functions, Nederl. Akad. Wetensch. Indag. Math. 41 (1979), no. 2, 105-110. MR 80j:41049

8. P. Flajolet and A. Odlyzko, Singularity analysis of generating functions, SIAM J. Discrete Math. 3 (1990), no. 2, 216-240. MR 90m:05012

9. M. Fukushima and T. Shima, On a spectral analysis for the Sierpiński gasket, Potential Anal. 1 (1992), no. 1, 1-35. MR 95b:31009

10. I. P. Goulden and D. M. Jackson, Combinatorial enumeration, John Wiley \& Sons, New York, 1983. MR 84m:05002

11. P. J. Grabner, Functional iterations and stopping times for Brownian motion on the Sierpinski gasket, Mathematika 44 (1997), no. 2, 374-400. MR 99b:60128

12. P. J. Grabner and W. Woess, Functional iterations and periodic oscillations for simple random walk on the Sierpiński graph, Stochastic Process. Appl. 69 (1997), no. 1, 127-138. MR 98h:60104

13. C. Inninger, Rational iteration, Universitätsverlag Rudolf Trauner, Linz, 2001, Dissertation, University of Linz, 2001. MR 2002b:37057

14. O. D. Jones, Transition probabilities for the simple random walk on the Sierpinski graph, Stochastic Process. Appl. 61 (1996), no. 1, 45-69. MR 97b:60115

15. J. Kigami, Harmonic calculus on p.c.f. self-similar sets, Trans. Amer. Math. Soc. 335 (1993), no. 2, 721-755. MR 93d:39008

16. _ Analysis on fractals, Cambridge Univ. Press, Cambridge, 2001. MR 2002c:28015 
17. J. Kigami and M. L. Lapidus, Weyl's problem for the spectral distribution of Laplacians on p.c.f. self-similar fractals, Comm. Math. Phys. 158 (1993), no. 1, 93-125. MR 94m:58225

18. B. Krön, Green functions on self-similar graphs and bounds for the spectrum of the Laplacian, Ann. Inst. Fourier 52 (2002), no. 6, 1875-1900.

19. - Growth of self-similar graphs, preprint, 2002.

20. T. Lindstrøm, Brownian motion on nested fractals, Mem. Amer. Math. Soc. 83 (1990), no. 420, iv+128. MR 90k:60157

21. L. Malozemov and A. Teplyaev, Pure point spectrum of the Laplacians on fractal graphs, J. Funct. Anal. 129 (1995), no. 2, 390-405. MR 96e:60114

22. Self-similarity, operators and dynamics, preprint, 2001.

23. A. M. Odlyzko, Periodic oscillations of coefficients of power series that satisfy functional equations, Adv. in Math. 44 (1982), no. 2, 180-205. MR 84a:30042

24. R. Rammal, Random walk statistics on fractal structures, J. Statist. Phys. 36 (1984), no. 5-6, 547-560. MR 85m:82118

25. R. Rammal and G. Toulouse, Random walks on fractal structures and percolation clusters, J. Physique Lettres 44 (1983), L13-L22.

26. C. Sabot, Spectral properties of hierarchical lattices and iteration of rational maps, Mem. Soc. Math. Fr. (N.S.) 92 (2003), vi + 104pp.

27. E. Teufl, The average displacement of the simple random walk on the Sierpinski graph, Combin. Probab. Comput. 12 (2003), 203-222.

28. W. Woess, Random walks on infinite graphs and groups, Cambridge University Press, Cambridge, 2000. MR 2001k:60006

Erwin Schrödinger Institute (ESI) Vienna, Boltzmanngasse 9, 1090 Wien, Austria

E-mail address: bernhard.kroen@univie.ac.at

Current address: Department of Mathematics, University of Vienna, Strudlhofgasse 4, 1090 Wien, Austria

Department of Mathematics C, Graz University of Technology, Steyrergasse 30 , 8010 Graz, Austria

E-mail address: elmar.teufl@tugraz.at 